\title{
Electromyographic Data Do Not Support a Progressive Recruitment of Muscle Fibers during Exercise Exhibiting a $\dot{\mathrm{V}}_{2}$ Slow Component
}

\author{
Daniel T. Cannon, Fred W. Kolkhorst and Daniel J. Cipriani \\ School of Exercise and Nutritional Sciences, San Diego State University, San Diego, CA, USA
}

\begin{abstract}
The origin of the slow component (SC) of oxygen uptake kinetics, presenting during exercise above the ventilatory threshold $\left(\mathrm{V}_{\mathrm{T}}\right)$, remains unclear. Possible physiologic mechanisms include a progressive recruitment of type II muscle fibers. The purpose of this study was to examine alterations in muscle activity through electromyography (EMG) and mean power frequency (MPF) analysis during heavy cycling exercise. Eight trained cyclists (mean \pm S.E.; age $=30 \pm 3$ years, height $=177 \pm 4 \mathrm{~cm}$, weight $=73.8 \pm 6.5 \mathrm{~kg}$, $\dot{\mathrm{V}}_{2 \max }=4.33 \pm 0.28 \mathrm{lmin}^{-1}$ ) completed transitions from $20 \mathrm{~W}$ to a workload equaling $50 \%$ of the difference between $\mathrm{V}_{\mathrm{T}}$ and $\dot{\mathrm{V}}_{2 \max }$. $\dot{\mathrm{VO}}_{2}$ was monitored using a breath-by-breath measurement system, and EMG data were gathered from surface electrodes placed on the gastrocnemius lateralis and vastus lateralis oblique. Breath-by-breath data were time aligned, averaged, interpolated to 1-s intervals, and modeled with non-linear regression. Mean power frequency (MPF) and RMS EMG values were calculated for each minute during the exercise bout. Additionally, MPF was determined using both isolated EMG bursts and complete pedal revolutions. All subjects exhibited a $\dot{\mathrm{VO}}_{2} \mathrm{SC}$ (mean amplitude $=0.98 \pm$ $0.16\left(\mathrm{~min}^{-1}\right)$, yet no significant differences were observed during the exercise bout in MPF or RMS EMG data $(p>0.05)$ using either analysis technique. While it is possible that the sensitivity of EMG may be insufficient to identify changes in muscle activity theorized to affect the $\dot{\mathrm{VO}}_{2} \mathrm{SC}$, the data indicated no relationship between MPF/EMG and the SC during heavy cycling. J Physiol Anthropol 26(5): 541-546, $2007 \mathrm{http}: / /$ www.jstage.jst.go.jp/browse/jpa2

[DOI: 10.2114/jpa2.26.541]
\end{abstract}

Keywords: EMG, cycling, oxygen uptake kinetics

\section{Introduction}

The kinetics of oxygen uptake for transitions from rest to work rate below the ventilatory threshold $\left(\mathrm{V}_{\mathrm{T}}\right)$ can be described with a first order exponential profile, resulting in a new steady state within 2 to 3 min (Whipp, 1987). Transitions to work rate above $\mathrm{V}_{\mathrm{T}}$ (termed heavy exercise) exhibit more complicated on-kinetics with the addition of a delayed, upward drift in oxygen consumption (Whipp and Rossiter, 2005). This characteristic of heavy exercise is identified as the slow component (SC). While there is certainly no conclusive evidence as to the origin of the SC, the vast majority of the "excess $\mathrm{V}_{2}$ " can be attributed to the exercising muscles, and not simply the rising cost of ventilation or cardiac output (Poole et al., 1991). When exercising at work rates above the $\mathrm{V}_{\mathrm{T}}$, it has been suggested the mechanistic basis of the SC may be alterations in muscle fiber recruitment pattern (Poole et al., 1994; Krustrup et al., 2004). More specifically, the fatigue of type I oxidative fibers during the first minutes of heavy intensity exercise may be followed by a progressive derecruitment of the fatiguing fibers, and recruitment of type IIa/x fibers (Whipp, 1987). As the anaerobic glycolytic fibers are less efficient (Crow and Kushmerick, 1982; Han et al., 2003) and more prone to fatigue, the SC may be attributed to this fall in economy and increased recruitment to maintain work output. In addition, subjects exhibiting high percentages of Type II fibers, or increased Type II recruitment after Type I glycogen reduction, show an exaggerated SC (Barstow et al., 1996; Pedersen et al., 2002; Jones et al., 2004; Berger et al., 2006). In contrast, Osborne and Schneider (2006) did not observe any augmentation in the SC amplitude with type I fiber glycogen reduction, while the phase I and II amplitudes were augmented. Phosphorous magnetic resonance spectroscopy $\left({ }^{31} \mathrm{P}\right.$ MRS), has shown inconclusive results in correlating inorganic phosphate shifts, characteristic of type II fiber recruitment (Yoshida and Watari, 1994), with the SC (Rossiter et al., 2002). Pringle and colleagues (2003) have also provided an alternate hypothesis whereas the type II fibers are initially recruited at the onset of heavy exercise, fatigue, and are supplemented with an up-regulation of type I oxidative fibers (Pringle et al., 2003; Krustrup et al., 2004). Evidence has yet to confirm either assertion.

Due to its non-invasive nature and relatively low cost, surface electromyography has been employed to examine these 
temporal changes in muscle fiber recruitment in a variety of exercising conditions. Electromyography (EMG), including the mean/median power frequency (MPF), are the tools most often used to examine motor unit recruitment and shifts in recruitment order. MPF is mostly determined by the mean conduction velocity of the muscle fiber, which is affected by cross sectional area and the type of muscle fiber (Loeb and Gans, 1986; Kupa et al., 1995). The measure of MPF has been employed on this basis to investigate fiber-recruitment adjustments during exercise (e.g. Bernasconi et al., 2006; Borrani et al., 2001; Garland et al., 2006; Komi et al., 2000; Osborne and Schneider, 2006; Sabapathy et al., 2005; Scheuermann et al., 2001).

During heavy and very-heavy exercise, research has shown increases in EMG and MPF associated with the $\dot{\mathrm{VO}}_{2} \mathrm{SC}$ (Burnley et al., 2002; Borrani et al., 2001; Bernasconi et al., 2006; Sabapathy et al., 2005). These finding support the theoretical basis for the SC arising largely from shifts in recruitment patterns. Yet others have reported no detectable increases in EMG or MPF suggesting a poor relationship between the SC and EMG data (Garland et al., 2006; Lucia et al., 2000; Pringle and Jones, 2002; Scheuermann et al., 2001).

Electromyographic evidence presented in the scientific literature remains unclear partly due to the inherent difficulties in data collection, analysis and interpretation involved with such measures. Furthermore, little consistency is found in determining MPF during dynamic exercise as it has often been analyzed not exclusively during muscle activation but during complete strides, standard time periods, or knee extension repetitions (Borrani et al., 2001; Garland et al., 2006; Osborne and Scneider, 2006). These results may have been confounded with an analysis of MPF during muscle inactivity, a period of muscular action which is an unlikely candidate for the origin of the SC. An alternative analysis of MPF using the EMG burst exclusively may provide better sensitivity to shifts in fiber recruitment type. Thus, the purpose of this study was to examine the EMG activity of the vastus lateralis oblique and the gastrocnemius lateralis during heavy cycling exercise in humans.

\section{Methods}

Eight trained cyclists agreed to participate in the investigation. Subject characteristics are presented in Table 1. Subjects were screened for cardiovascular risk via the Physical Activity Readiness Questionnaire (PAR-Q). Written informed consent was obtained from all volunteers before participating. The study protocol was approved by the San Diego State University Institutional Review Board. All procedures complied with the Declaration of Helsinki.

\section{Maximal oxygen consumption and ventilatory threshold}

Volunteers reported to the laboratory for a maximally graded exercise test on an electromagnetically braked cycle ergometer (Lode Excalibur, Lode BV, Groningen, The Netherlands). The exercise test consisted of a 3 min warm-up at a power of $50 \mathrm{~W}$, followed by a ramp protocol applied at a rate of $25 \mathrm{~W} \mathrm{~min}{ }^{-1}$ until subjects reached volitional exhaustion. Expired gases were analyzed breath-by-breath (VMax Encore, Viasys Healthcare, Yorba Linda, CA, USA). Maximal oxygen consumption $\left(\dot{\mathrm{V}}_{2 \max }\right)$ was achieved according to the following criteria: subjects unable to maintain a pedal cadence of $60 \mathrm{rpm}, \mathrm{RER} \geq 1.1$, maximal heart rate $\left(\mathrm{HR}_{\max }\right)$ within $10 \mathrm{bpm}$ of age-predicted maximum. All subjects received verbal encouragement until exhaustion. $\dot{\mathrm{VO}}_{2 \max }$ was determined from the highest $20 \mathrm{~s}$ average. Ventilatory threshold $\left(\mathrm{V}_{\mathrm{T}}\right)$ was determined by multiple criteria; including a rapid increase in the ventilatory equivalent of oxygen $\left(\dot{\mathrm{V}}_{\mathrm{E}} / \dot{\mathrm{VO}}_{2}\right)$ with no concomitant increase in ventilatory equivalent of carbon dioxide $\left(\dot{\mathrm{V}}_{\mathrm{E}} / \dot{\mathrm{VCO}}_{2}\right)$, as well as the V-slope method (Beaver et al., 1986).

\section{Exercise protocol and oxygen uptake kinetics measurement/ analysis}

Subjects returned to the laboratory on three occasions, separated by at least 24 hours each, to complete square wave transitions to heavy cycling exercise. Cycling work rate equaled $50 \%$ of the difference between $\mathrm{V}_{\mathrm{T}}$ and $\dot{\mathrm{VO}}_{2 \max }$ $(50 \% \Delta)$. The protocol consisted of the subjects pedaling at $20 \mathrm{~W}$ for $6 \mathrm{~min}$ with an immediate transition to a power equal to $50 \% \Delta$, lasting $6 \mathrm{~min}$. Participants were instructed to pedal at a rate of $90 \mathrm{rpm}$, and were provided with a digital display to

Table 1 Subject characteristics

\begin{tabular}{ccccccc}
\hline Subject & Sex & Age & Height $(\mathrm{cm})$ & Weight $(\mathrm{kg})$ & $\dot{\mathrm{VO}}_{2 \max }\left(l \mathrm{~min}^{-1}\right)$ & $\dot{\mathrm{VO}}_{2 @} \mathrm{~V}_{\mathrm{T}}\left(l \mathrm{~min}^{-1}\right)$ \\
\hline 1 & $\mathrm{M}$ & 23 & 193 & 113.6 & 5.433 & 3.757 \\
2 & $\mathrm{~F}$ & 41 & 165 & 59.1 & 3.115 & 2.010 \\
3 & $\mathrm{M}$ & 23 & 183 & 82.7 & 5.147 & 3.405 \\
4 & $\mathrm{M}$ & 21 & 175 & 68.2 & 4.454 & 3.062 \\
5 & $\mathrm{M}$ & 35 & 170 & 63.6 & 4.506 & 3.942 \\
6 & $\mathrm{M}$ & 31 & 173 & 60.0 & 4.447 & 2.024 \\
7 & $\mathrm{~F}$ & 40 & 170 & 63.6 & 3.440 & 2.124 \\
8 & $\mathrm{M}$ & 33 & $177 \pm 4$ & $73.8 \pm 6.5$ & $4.33 \pm 0.28$ & $2.91 \pm 0.20$ \\
\hline
\end{tabular}


monitor pedal cadence.

Breath-by-breath data were visually inspected in order to eliminate non-physiologic data points resulting from aberrant breaths (i.e. cough, swallow, hiccup etc.). Data were then filtered to eliminate points lying outside of $3 \mathrm{SD}$ in respect to an $8 \mathrm{~s}$ rolling average. Data were time aligned, combined, averaged, and interpolated to $1-\mathrm{sec}$ intervals. The first $20 \mathrm{~s}$ of the exercise bout were eliminated to circumvent the cardiopulmonary (phase I) component (Whipp and Rossiter, 2005). A biexponential function was fitted to the data according to the equation below, whereas $\mathrm{VO}_{2(\mathrm{t})}$ is the timedependent variation of $\dot{\mathrm{VO}}_{2}$. The model included a baseline term $\left(\dot{\mathrm{V}}_{2 \text { base }}\right)$, two asymptotic amplitude terms $\left(\mathrm{A}_{\mathrm{p}}, \mathrm{A}_{\mathrm{s}}\right)$, two time constants $\left(\tau_{\mathrm{p}}, \tau_{\mathrm{s}}\right)$, and two time delays $\left(\mathrm{TD}_{\mathrm{p}}, \mathrm{TD}_{\mathrm{s}}\right)$.

$$
\dot{\mathrm{V}} \mathrm{O}_{2 \mathrm{t}}=\dot{\mathrm{V}} \mathrm{O}_{2 \text { base }}+\mathrm{A}_{\mathrm{p}} \cdot\left(1-\mathrm{e}^{-\left(t-\mathrm{TD}_{\mathrm{p}}\right) / \tau_{\mathrm{p}}}\right)+\mathrm{A}_{\mathrm{s}} \cdot\left(1-\mathrm{e}^{-\left(t-\mathrm{TD}_{\mathrm{s}}\right) / \tau_{\mathrm{s}}}\right)
$$

\section{Electromyography}

One of the three laboratory visits were randomly chosen to collect EMG data from the gastrocnemius lateralis and vastus lateralis obligue. The two muscles were chosen on the basis of opposing muscular action (Ericson, 1988) and to provide measurement of muscle mass with predominantly different fiber type distributions. Electrode placement sites were shaved, abraded with gauze, and cleaned with alcohol. Bipolar $\mathrm{Ag} / \mathrm{AgCl}$ pre-gelled surface electrodes with an inter-electrode distance of $2 \mathrm{~cm}$ were placed over the appropriate muscle belly. The conductive area of each electrode was $1 \mathrm{~cm}$ in diameter and each bipolar electrode used a proximal differential amplifier. Electrodes were placed on the vastus lateralis oblique halfway between the greater trochanter and the patella and on the gastrocnemius lateralis just distal from the midpoint between the popliteal fossa and the musculotendinous junction. The longitudinal axis of the electrodes was aligned parallel to the muscle fibers and a reference electrode was placed on the head of the fibula. Electrodes were placed on each subject by the same investigator.

The incoming EMG signals were pre-amplified with a gain of 500 and included a channel filter of a first order high-pass filter at $20 \mathrm{~Hz}$ and an eighth order Butterworth/Bessels low pass anti-alias filter set at $500 \mathrm{~Hz}$. The signal was digitized at $1,000 \mathrm{~Hz}$ (Noraxon Telemyo 2400T, Noraxon USA, Scottsdale AZ, USA). Data were collected for approximately $5 \mathrm{~s}$ at $50 \mathrm{~s}$ into each minute of exercise to provide data for min 1-6.

For mean power frequency analysis, three bursts of EMG were visually identified and randomly sampled from each $5 \mathrm{~s}$ period of data collection. MPF (Hz) was determined via power spectral density function and fast Fourier transformation. Additionally, MPF analysis was completed using 3 complete pedal revolutions to identify any differences due to analysis technique. To estimate muscular activity, the signal was full wave rectified and smoothed with a $50 \mathrm{~ms}$ moving window, and three complete pedal revolutions were sampled and analyzed via RMS to provide EMG data $(\mu \mathrm{V})$ (MyoResearch $\mathrm{XP}$, Noraxon USA, Scottsdale AZ, USA).
Statistical analysis

Descriptive statistics were generated for subject characteristics as and oxygen uptake kinetics (mean \pm S.E.). Repeated measures ANOVA was used to examine differences in MPF and EMG across each of the 6 time periods. Bonferroni corrected $(\alpha=\alpha / \kappa$, with $\kappa$ equal to number of comparisons) paired t-tests were used to compare analysis techniques (EMG bursts vs. full pedal revolutions) at each time period. Data were analyzed using the Statistical Package for the Social Sciences (SPSS v13.0, SPSS Inc., Chicago, IL, USA). Statistical significance was determined at $p<0.05$. All data are presented as means \pm S.E.

\section{Results}

Mean $\dot{\mathrm{VO}}_{2 \max }$ achieved during the ramped test was $4.33 \pm$ $0.28 \mathrm{lmin}^{-1}$, at a work rate of $361 \pm 16 \mathrm{~W}$. $\mathrm{V}_{\mathrm{T}}$ was reached at a mean $\dot{\mathrm{VO}}_{2}$ of $2.91 \pm 0.20 \mathrm{lmin}^{-1}$ at a work rate of $240 \pm 24 \mathrm{~W}$ $\left(67 \pm 1.0 \% \dot{\mathrm{VO}}_{2 \max }\right)$. During the $6 \mathrm{~min}$ square-wave transitions to exercise, work rate at $50 \% \Delta$ was $286 \pm 15 \mathrm{~W}$. The $\mathrm{VOO}_{2}$ kinetic responses are presented in Table 2. All subjects exhibited a SC during phase III indicative and expected of the heavy exercise domain (Fig. 1, Table 2). No significant differences were found for EMG of the gastrocnemius $\left[\mathrm{F}(5,35)=1.065, \quad p>0.05, \quad \eta^{2}=0.132\right] \quad$ or vastus lateralis oblique $\left[\mathrm{F}(1.949,13.646)=1.494, p>0.05, \eta^{2}=0.176\right]$. No significant differences were found for MPF of the gastrocnemius $\left[\mathrm{F}(1.455,10.186)=0.831, p>0.05, \quad \eta^{2}=0.106\right] \quad$ or vastus lateralis oblique $\left[\mathrm{F}(5,35)=1.780, p>0.05, \eta^{2}=0.203\right]$ (Figs. $2,3,4)$ when complete pedal revolutions were analyzed. Additionally, no differences were found in MPF when only

Table $2 \quad \mathrm{VO}_{2}$ kinetic parameters during transition from $20 \mathrm{~W}$ to a work rate equal to $50 \% \Delta$

\begin{tabular}{cc}
\hline Parameter & mean6S.E. \\
\hline Phase II Amplitude $\left(l \mathrm{~min}^{-1}\right)$ & $2.46 \pm 0.12$ \\
Phase II Time Constant $(t)(\mathrm{s})$ & $13.1 \pm 1.8$ \\
Phase II Time Delay (TD) (s) & $9.4 \pm 2.0$ \\
Phase III Amplitude $\left(l \mathrm{~min}^{-1}\right)$ & $0.98 \pm 0.16$ \\
Phase III Time Constant $(t)(\mathrm{s})$ & $218 \pm 102$ \\
Phase III Time Delay (TD) (s) & $90.0 \pm 14.8$ \\
\hline
\end{tabular}

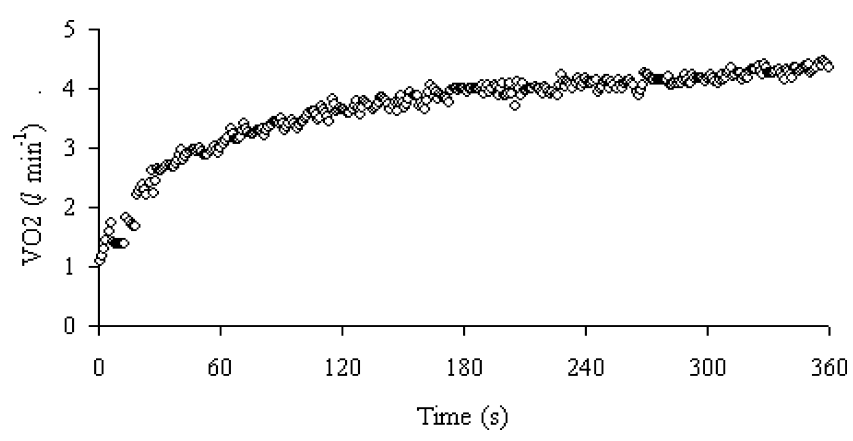

Fig. 1 Representative subject $\mathrm{V}_{2}$ response during 6 min heavy exercise bout. 


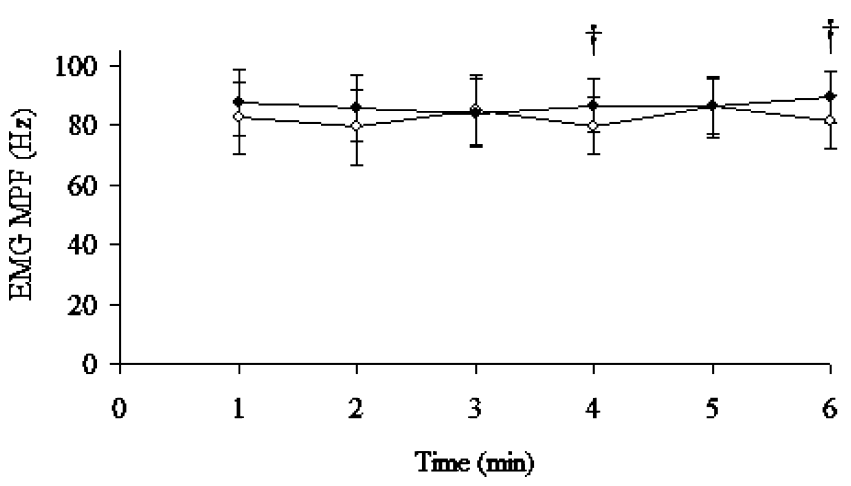

Fig. 2 Mean power frequency (MPF) of the gastrocnemius lateralis during $6 \mathrm{~min}$ heavy cycling bout. Open symbols represent MPF analysis of 3 EMG bursts, closed symbols represent MPF analysis of 3 pedal revolutions. $\dagger$ denotes significant difference between analysis technique $(p<0.05)$.

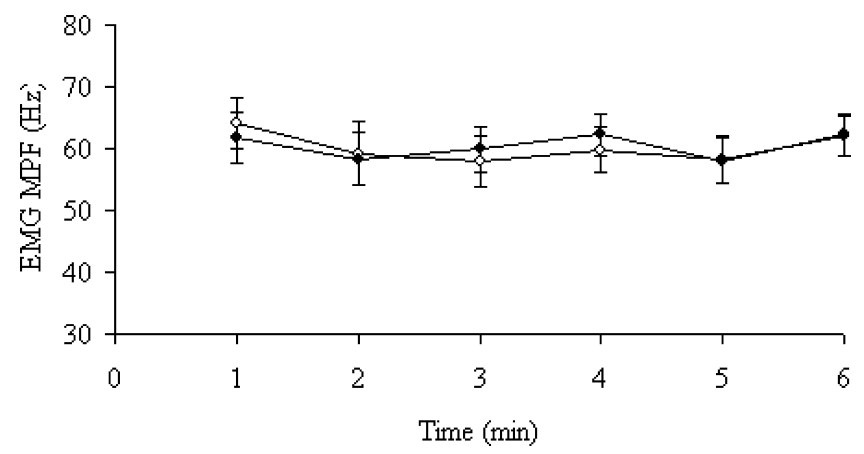

Fig. 3 Mean power frequency (MPF) of the vastus lateralis oblique during 6 min heavy cycling bout. Open symbols represent MPF analysis of 3 EMG bursts, closed symbols represent MPF analysis of 3 pedal revolutions.

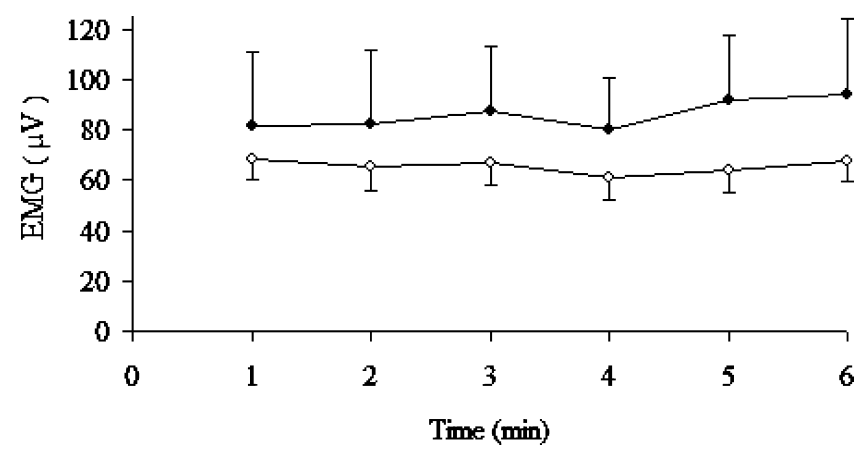

Fig. 4 EMG during 6 min heavy cycling bout. Open symbols represent gastrocnemius lateralis, closed symbols represent vastus lateralis oblique.

EMG bursts were sampled for the gastrocnemius $[\mathrm{F}(1.912$, 13.382) $=0.345, p>0.05, \eta^{2}=0.047$ ] or vastus lateralis oblique $\left[\mathrm{F}(5,35)=1.334, p>0.05, \eta^{2}=0.160\right]$. Paired t-tests revealed significantly higher MPF values at $\min 4\left(p<0.008 ; \mathrm{CI}_{\Delta} 2.77\right.$, $10.77)$ and $\min 6\left(p<0.008 ; \mathrm{CI}_{\Delta} 2.17,14.19\right)$ for the gastrocnemius lateralis when comparing analysis techniques (Fig. 2). No significant differences were observed between the analysis techniques at the vastus lateralis oblique $(p>0.05)$.

\section{Discussion}

The primary finding of this investigation was an absence of electromyographic evidence for a progressive recruitment of type IIa/x fibers during a heavy exercise bout exhibiting a SC. While the data indicate the possibility that analyzing complete pedal revolutions can alter MPF analyses, as compared to analyzing EMG bursts alone, the changes were not marked enough to suggest an alternate conclusion as to changes in motor unit recruitment during heavy exercise, nor were the findings of physiologic or practical significance (Fig. 2). This indicated that the sampling period including muscle inactivity did not measurably affect the MPF during dynamic exercise. While muscle inactivity during dynamic exercise is of comparatively little interest, in regards to the bioenergetics, these data indicate that sampling periods including complete pedal revolutions can provide reliable MPF data, and that either are appropriate for analysis. These data suggested that either alteration in fiber recruitment was not present, or that factors intrinsic to the initially recruited muscle fibers may have been responsible for the SC. The data expounded in the present investigation are in agreement with the assertions that the SC originates from intrinsic factors to the initially recruited fibers (Garland et al., 2006; Lucia et al., 2000; Pringle and Jones, 2002; Scheuermann et al., 2001).

These factors include reductions in metabolic efficiency due to an elevated muscle temperature (Koga et al., 1997), or disturbances to creatine kinase from an acidosis incurred during heavy exercise (Kushmerick, 1998). While ion handling during heavy exercise has not been examined experimentally in relation to the development of the $\mathrm{SC}$, the disruption in $\mathrm{Ca}^{++} / \mathrm{K}^{+} / \mathrm{Na}^{+}$ATPase activity has been suggested by Scheuermann and co-workers (2001) as a possible contributor to the SC. Investigations have shown marked alterations in sarcoplasmic reticulum calcium uptake and release after bouts of repetitive muscular contractions (Duhamel et al., 2004; Booth et al., 1997; Tupling et al., 2003; Li et al., 2002), providing experimental evidence to support these hypotheses.

While the recruitment of type IIa/x fibers remains a popular theory to explain the $\mathrm{SC}$, the force production during heavy or even severe cycling does not typically approach the thresholds of muscle force output typically associated with the recruitment of type II fibers. During high intensity cycling, values ranging from $\sim 11-20 \% \mathrm{MVC}$ are common (Löllgen et al., 1980; St. Clair Gibson et al., 2001). The data in the present investigation support the notion that muscle effort during heavy cycling does not result in progressive recruitment of type II muscle fibers. It is unlikely that an appreciable number of type I muscles fibers fatigued during the exercise bout in the present investigation; negating the need for a progressive recruitment of the anaerobic glycolytic fibers. While Garland and associates (2006) emphasized the assignment of work rate well below the critical power (CP) threshold when examining 
the SC to avoid rapid muscle fiber fatigue, the work rate chosen for this investigation may have been at or above CP, but failed to produce a change in either electromyographic measure.

The lack of temporal change in MPF and EMG cannot rule out a fiber recruitment hypothesis as a mechanistic basis for the SC. It is a possibility that these findings may have resulted from a lack of sensitivity required to identify relatively small changes in motor unit recruitment. By using glycogen depletion to preferentially select type II fiber recruitment, Osborne and Schneider (2006) reported $\sim 4 \%$ change in recruitment of type II fibers (via equation of Wretling) based on significant findings of increasing MPF. These investigators speculated that the lack of augmentation to the slow phase with increases in MPF may be due to the relatively small physiologic significance of the shift in recruitment. Muscle fiber recruitment patterns may have changed throughout the exercise bout in the present study, but due to the push-pull factors discussed by Garland and associates (2006), these changes may not be identified by the measure of MPF or EMG. It has been reported that fatiguing type IIa/x fibers may be replaced by proportionally more type I fibers, which are less efficient at high power output and generate less force (Garland et al., 2006; He et al., 2000). The net result could possibly be unchanging EMG and MPF data (Garland et al., 2006). Additionally, as type II muscle fibers are recruited, the expected rise in MPF may be offset by a synchronization of said muscle fibers, which will lead to a shift downward in MPF (Loeb and Gans, 1986). Furthermore, the net loss of $\mathrm{K}^{+}$ resulting from muscular contraction may blunt the propagation of action potentials across the cell membrane and reduce MPF (Gamet et al., 1993). A major limitation of this study is the inability to elucidate these physiologic factors that may have influenced the results. Currently no measure exists to isolate these phenomenons from a true, "static" MPF resulting from an unchanging recruitment pattern.

In conclusion, the data presented have indicated no association between the development of the SC and a shift in EMG and MPF during heavy cycling exercise. While the complexities of the electromyographic measurement may have influenced the ability to elucidate small changes in muscle activity, the data remain in conflict with the assertion that the $\mathrm{SC}$ arises from the progressive recruitment of the type IIa/x muscle fibers and/or an increase in recruitment of type I fibers.

\section{References}

Barstow TJ, Jones AM, Nguyen P, Casaburi R (1996) Influence of muscle fiber type and pedal frequency on oxygen uptake kinetics of heavy exercise. J Appl Physiol 75: 755-762

Beaver WL, Wasserman K, Whipp BJ (1986) A new method for detecting anaerobic threshold by gas exchange. J Appl Physiol 60: 2020-2027

Berger NJ, Rittweger J, Kwiet A, Michaelis I, Williams AG, Tolfrey K, Jones AM (2006) Pulmonary $\mathrm{O}_{2}$ Uptake On-
Kinetics in Endurance- and Sprint-Trained Master Athletes. Int J Sports Med 27: 1005-1012

Bernasconi S, Tordi N, Perrey S, Parrantte B, Monnier G (2006) Is the $\mathrm{VO}_{2}$ slow component in heavy arm-cranking exercise associated with recruitment of type II muscle fibers as assessed by an increase in surface EMG? Appl Physiol Nutr Metab 31: 414-422

Booth J, McKenna MJ, Ruell PA, Gwinn TH, Davis GM, Thompson MW, Harmer AR, Hunter SK, Sutton JR (1997) Impaired calcium pump function does not slow relaxation in human skeletal muscle after prolonged exercise. J Appl Physiol 83: 511-521

Borrani F, Candau R, Millet GY, Perrey S, Fuchslocher J, Rouillon JD (2001) Is the $\dot{\mathrm{VO}_{2}}$ slow component dependent on progressive recruitment of fast-twitch fibers in trained runners? J Appl Physiol 90: 2212-2220

Burnley M, Doust JH, Ball D, Jones AM (2002) Effects of prior heavy exercise on $\mathrm{V}_{2}$ kinetics during heavy exercise are related to changes in muscle activity. J Appl Physiol 93: 167-174

Crow MT, Kushmerick MJ (1982) Chemical energetics of slow and fast twitch muscles of the mouse. J General Physiol 79: 147-166

Duhamel TA, Green HJ, Perco JD, Sandiford SD, Ouyang J (2004) Human muscle sarcoplasmic reticulum function during submaximal exercise in normoxia and hypoxia. J Appl Physiol 97: 180-187

Ericson MO (1988) Muscular function during ergometer cycling. Scand J Rehab Med 20: 35-41

Gamet D, Duchene J, Garapon-Bar C, Goubel F (1993) Surface electromyogram power spectrum in human quadriceps muscle during incremental exercise. J Appl Physiol 74: 2704-2710

Garland SW, Wang W, Ward SA (2006) Indices of electromyographic activity and the "slow component" of oxygen uptake kinetics during high-intensity knee-extension exercise in humans. Eur J Appl Physiol 94: 413-423

Han YS, Geiger PC, Cody MJ, Macken RK, Sieck GC (2003) ATP consumption rate per cross bridge depends on myosin heavy chain isoforms. J Appl Physiol 94: 2188-2196

He ZH, Bottinelli R, Pellegrino MA, Ferenczi MA, Reggiani C (2000) ATP consumption and efficiency of human single muscle fibers with different myosin isoforms composition. Biophys J 76: 945-961

Jones AM, Campbell IT, Pringle JSM (2004) Influence of muscle fiber type and pedal rate on the $\mathrm{VO}_{2}$ work rate slope during ramp exercise. Eur J Appl Physiol 91: 238-245

Koga S, Shiojiri T, Konda N, Barstow TJ (1997) Effect of increased muscle temperature on oxygen uptake kinetics during exercise. J Appl Physiol 83: 1333-1338

Komi PV, Linnamo V, Silventoinen P, Sillanpää M (2000) Force and EMG power spectrum during eccentric and concentric actions. Med Sci Sports Exerc 32: 1757-1762

Krustrup P, Soderlund K, Mohr M, Bangsbo J (2004) The slow component of oxygen uptake during intense, sub-maximal 
exercise in man is associated with additional fiber recruitment. Pflugers Arch 447: 855-866

Kupa EJ, Roy SH, Kandarian SC, DeLuca CJ (1995) Effects of muscle fiber type and size on EMG median frequency and conduction velocity. J Appl Physiol 79: 23-32

Kushmerick MJ (1998) Energy balance in muscle activity: Simulations of ATPase coupled to oxidative phosphorylation and to creatine kinase. Comp Biochem Physiol B 120: 109-123

Loeb GE, Gans C (1986) Electromyography for Experimentalists. The University of Chicago Press, Chicago and London, 258

Lollgen H, Graham T, Sjogaard G (1980) Muscle metabolites, force, and perceived exertion bicycling at varying pedal rates. Med Sci Sports Exerc 12: 345-351

Li JL, Wang XN, Fraser SF, Carey MF, Wrigley TV, McKenna MJ (2002) Effects of fatigue and training on sarcoplasmic reticulum $\mathrm{Ca}^{2+}$ regulation in human skeletal muscle. J Appl Physiol 92: 912-922

Lucia A, Hoyos J, Chicharro JL (2000) The slow component of $\dot{\mathrm{V}} \mathrm{O}_{2}$ in professional cyclists. Br J Sports Med 34: 367-374

Osborne MA, Schneider DA (2006) Muscle glycogen reduction in man: relationship between surface EMG activity and oxygen uptake kinetics during heavy exercise. Exp Physiol 91: 179-189

Pedersen PK, Sorensen JB, Jensen K, Johansen L, Levin K (2002) Muscle fiber type distribution and nonlinear $\dot{\mathrm{V}} \mathrm{O}_{2}$ power output relationship in cycling. Med Sci Sports Exerc 34: 655-661

Poole DC, Schaffartzik W, Knight DR, Derion T, Kennedy B, Guy HJ, Prediletto R, Wagner PD (1991) Contribution of exercising legs to the slow component of oxygen uptake kinetics in humans. J Appl Physiol 71: 1245-1260

Poole DC, Barstow TJ, Gaesser GA, Willis WT, Whipp BJ (1994) $\dot{\mathrm{VO}}_{2}$ slow component: physiological and functional significance. Med Sci Sports Exerc 26: 1354-1358

Pringle JSM, Jones AM (2002) Maximal lactate steady state, critical power and EMG during cycling. Eur J Appl Physiol 88: 214-226

Pringle JSM, Doust JH, Carter H, Tolfrey K, Jones AM (2003) Effect of pedal rate on primary and slow component oxygen uptake responses during heavy-cycle exercise. J Appl Physiol 94: 1501-1507

Rossiter HB, Ward SA, Howe FA, Kowalchuk JM, Griffiths JR, Whipp BJ (2002) Dynamics of intramuscular P-31-MRS Pi peak splitting and the slow components of $\mathrm{PCr}$ and $\mathrm{O}_{2}$ uptake during exercise. J Appl Physiol 93: 2059-2069

Sabapathy S, Schneider DA, Morris NR (2005) The $\dot{\mathrm{VO}}_{2}$ slow component: relationship between plasma ammonia and EMG activity. Med Sci Sports Exerc 37: 1502-1509

Scheuermann BW, Hoetling BD, Noble ML, Barstow TJ (2001) The slow component of $\mathrm{O}_{2}$ uptake in not accompanied by changes in muscle EMG during repeated bouts of heavy exercise in humans. J Physiol 531: 245-256

St. Clair Gibson A, Schabort EJ, Noakes TD (2001) Reduced neuromuscular activity and force generation during prolonged cycling. Am J Physiol Regul Integr Comp Physiol 281: $187-196$

Tupling R, Green HJ, Roy BD, Grant S, Ouyang J (2003) Paradoxical effects of prior activity on human sarcoplasmic reticulum $\mathrm{Ca}^{2+}$-ATPase response to exercise. J Appl Physiol 95: $138-144$

Whipp BJ (1987) Dynamics of pulmonary gas exchange. Circulation 76 (Suppl 5): 1-18

Whipp BJ, Rossiter HB (2005) The kinetics of oxygen uptake: physiological inferences from the parameters. In Jones AM, Poole DC eds. Oxygen Uptake Kinetics in Sport, Exercise, and Medicine. Routledge, London, 64-94

Yoshida T, Watari H (1994) Exercise-induced splitting of the inorganic phosphate peak: investigation by time-resolved 31P-nuclear magnetic resonance spectroscopy. Eur J Appl Physiol Occup Physiol 69: 465-473

Received: April 27, 2007

Accepted: July 27, 2007

Correspondence to: Daniel T. Cannon, School of Exercise and Nutritional Sciences San Diego State University 5500 Campanile Drive San Diego, CA 92182-7251, USA

Phone: 619-594-4459

Fax: 619-594-6553

e-mail: dcannon@rohan.sdsu.edu 